\title{
A relevância da implantação do Centro de Referência do Pé Diabético em Marabá-
} PA

\author{
The relevance of implementing a Reference Center on Diabetic Foot in Marabá-PA, Brazil \\ La relevancia de la implantación del centro de referencia del pie diabético en Marabá-PA
}

Recebido: 29/04/2021 | Revisado: 06/05/2021 | Aceito: 09/05/2021 | Publicado: 22/05/2021

\author{
Tatiana Teixeira de Castro Carvalho Beckemkamp \\ ORCID: https://orcid.org/0000-0003-1960-7702 \\ Universidade do Estado do Pará, Brasil \\ E-mail: tativascular@hotmail.com \\ Sandro Percário \\ ORCID: https://orcid.org/0000-0002-9528-0361 \\ Universidade Federal do Pará, Brasil \\ E-mail: percario@ufpa.br
}

\begin{abstract}
Resumo
Objetivo: Demonstrar a importância da criação do Centro de Referência do Pé Diabético de Marabá (CRPDM) para a cidade e região. Metodologia: Trata-se de um estudo transversal, observacional, retrospectivo conduzido em um hospital geral do Município de Marabá-Pa. A população do estudo correspondeu aos pacientes atendidos pela cirurgia vascular, no Hospital Municipal de Marabá, no período entre abril de 2015 a setembro de 2019. Resultados: Verificou-se um número elevado de atendimentos do serviço de cirurgia vascular no período estudado. Foram realizados 621 atendimentos com predominância de procedimentos cirúrgicos (51\%), entre amputações e revisões de coto, e pacientes com diagnóstico de diabetes. Em relação ao gênero houve uma prevalência do sexo masculino, quanto ao município de origem $85 \%$ eram de Marabá. Conclusão: Nessa perspectiva, o CRPDM tem como objetivo implantar um serviço de referência para os pacientes diabéticos, baseado nos protocolos e recomendações do Ministério da Saúde, Sociedade Brasileira de Diabetes - SBD e International Working Group on the Diabetic Foot IWGDP. A perspectiva é mudar a realidade da cidade e região de Marabá, promovendo melhorias na assistência, com o intuito de diminuir as complicações e sequelas nessa população.
\end{abstract}

Palavras-chave: Pé diabético; Centro de prevenção; Diabetes mellitus.

\begin{abstract}
Objective: Demonstrating the importance of the creation of Marabá Diabetic Foot Reference Center (CRPDM in Portuguese) for the city and region. Methodology: This is a cross-sectional, observational, retrospective study conducted at a general hospital in the city of Marabá-PA, Brazil. The study population is consisted of patients treated by the Vascular Surgery Department at the Hospital Municipal de Marabá, from April 2015 to September 2019. Results: It was identified a high number of healthcare services in the period in the department of vascular surgery. A total of 621 services were provided with the predominance of surgical procedures $(51 \%)$, including amputations and stump revisions and patients diagnosed with diabetes. Regarding gender there was a male prevalence and considering origin $85 \%$ of the patients were from Marabá. Conclusion: In view of that, the CRPDM aims to implement a reference service for diabetic patients, based on the protocols and recommendations of the Ministério da Saúde (Federal Health Ministry), Sociedade Brasileira de Diabetes - SBD (Brazilian Diabetes Society), and International Working Group on the Diabetic Foot (IWGDF). The perspective is to change the reality of the city and region of Marabá, promoting improvements in care to reduce complications and sequelae in this population.
\end{abstract}

Keywords: Diabetic foot; Prevention center; Diabetes mellitus.

\section{Resumen}

Objetivo: demostrar la importancia de la creación del Centro de Referencia del Pie Diabético de Marabá (CRPDM) para la ciudad y la región. Metodología: se trata de un estudio transversal, observacional, retrospectivo conducido en un hospital general del Municipio de Marabá-Pa. La población del estudio se corresponde con los pacientes atendidos por el departamento de cirugía vascular, en el Hospital Municipal de Marabá, en el período entre abril de 2015 y septiembre de 2019. Resultados: se verificó un número elevado de consultas del servicio de cirugía vascular en el período estudiado. Fueron realizadas 621 consultas con predominio de procedimientos quirúrgicos (51\%), entre amputaciones y revisiones de muñones, y pacientes con diagnóstico de diabetes. En relación al género hubo un predominio del sexo masculino, en cuanto al municipio de origen $85 \%$ eran de Marabá. Conclusión: en esta perspectiva, el CRPDM tiene como objetivo implantar un servicio de referencia para los pacientes diabéticos, basado en los protocolos y recomendaciones del Ministerio de Salud, la Sociedad Brasileña de Diabetes - SBD y el International Working Group on the Diabetic Foot - IWGDP. La perspectiva es mudar la realidad de la ciudad y la 
región de Marabá, promoviendo mejoras en la asistencia, con el propósito de disminuir las complicaciones y secuelas en esta población.

Palabras clave: Pie diabético; Centro de prevención; Diabetes Mellitus.

\section{Introdução}

Diabetes mellitus (DM) é um importante e crescente problema de saúde para todos os países, independentemente do seu grau de desenvolvimento (Gonçalves \& Moura, 2019). No Brasil, o diagnóstico dessa enfermidade aumentou 61,8\% em 10 anos. Entre 2006 e 2016, o número de pessoas que dizem saber do diagnóstico de diabetes passou de 5,5\% para 8,9\%. As mulheres lideram esse ranking, sendo que $9,9 \%$ da população feminina declarou possuir a doença contra apenas 7,8\% dos homens (Brasil, 2017).

O diabetes está entre as doenças crônicas mais prevalentes do século XXI, sendo caracterizado por um distúrbio metabólico em que ocorre hiperglicemia crônica e alterações do metabolismo de carboidratos, proteínas e gorduras, sendo decorrentes de defeitos da secreção e/ou ação da insulina (Karami-Mohajeri \& Abdollahi, 2011).

De acordo com estudos, aproximadamente 400 milhões de indivíduos no mundo possuem diabetes, e no ano de 2035 haverá 600 milhões de diabéticos (Karami-Mohajeri \& Abdollahi, 2011).

Diversos fatores estão relacionados ao aumento da prevalência do diabetes, como o estilo de vida sedentário, o excesso de peso, mudança nutricional e o crescimento e envelhecimento da população (Brasil, 2014).

As complicações do diabetes diferenciam-se em microvasculares e macrovasculares, sendo o pé diabético uma complicação microvascular e a mais devastadora. Está relacionada com infecção e úlceras, atingindo até os músculos e ossos, envolvendo anormalidades neurológicas periféricas de graus variados. A fisiopatologia do pé diabético origina-se da junção da neuropatia com a vasculopatia (Girach et al., 2006).

Adicionalmente, muitos pacientes não seguem o tratamento corretamente, ficando com a glicemia descompensada e gerando diversas complicações que podem ser reversíveis e irreversíveis, entre elas o pé diabético, que quando não é cuidado e tratado precocemente, pode evoluir até para amputações dos membros inferiores (Santos, 2017).

As amputações de membros inferiores são complicações irreversíveis e drásticas, sendo seu risco influenciado por vários fatores (descontrole glicêmico, descontrole pressórico, tabagismo, etc.) e depende da habilidade dos sistemas de saúde em rastrear o risco, estratificá-lo e tratar os pés de alto risco e as úlceras (Silveira et al., 2017).

Indivíduos portadores de diabetes apresentam uma incidência anual de $2 \%$ a $25 \%$ ao longo da vida de apresentarem úlceras nos pés. Aproximadamente 20\% das internações hospitalares são decorrentes de lesões de membros inferiores. Dados ainda mais graves apontam que as complicações decorrentes do pé diabético são responsáveis por $40 \%$ a $70 \%$ do total de amputações não traumáticas de membro inferiores na população em geral (Brasil, 2016).

Um estudo conduzido em um hospital geral de ensino e pesquisa, especializado em média e alta complexidade, da região metropolitana de Goiânia, apontou uma incidência de 61,9\% de amputações em pacientes diabéticos hospitalizados com uma prevalência de indivíduos do sexo masculino e ainda, o tipo de pé diabético mais frequente na amostra geral e nos amputados foi o neuropático (Oliveira et al., 2016).

No intuito de modificar essa realidade foram criadas em diversas capitais brasileiras os Centros de Referência para pacientes com pé diabético, com foco no diagnóstico precoce e prevenção de lesões graves, prevenindo sequelas como as amputações que limitam e diminuem a qualidade de vida do indivíduo (Brasil, 2016).

A organização da rede de atenção, assim como o treinamento de profissionais de saúde demonstram benefícios na estratificação de risco e intervenções dos pacientes com lesões de pé diabético, além de promover redução das amputações quando comparado aos cuidados convencionais (Brasil, 2016). 
Diante da necessidade da região e com a intenção de abranger o sudeste paraense, o Hospital Municipal de Marabá (HMM) será a referência para esses pacientes, após a criação do Centro de Referência do Pé Diabético de Marabá (CRPDM), através de parcerias da prefeitura de Marabá e do Mestrado Profissional de Cirurgia e Pesquisa Experimental (CIPE) da Universidade do Estado do Pará (UEPA).

Nesse cenário, o presente trabalho tem como objetivo demonstrar a importância da criação do Centro de Referência do Pé Diabético de Marabá, para a cidade e região.

\section{Metodologia}

Trata-se de um estudo transversal, observacional, retrospectivo, de natureza quantitativa (Estrela, 2018), conduzido em um hospital geral, o Hospital Municipal de Marabá (HMM), especializado em média complexidade, sendo a unidade de referência para 22 municípios da região do Sudeste do Pará.

A população do estudo correspondeu aos pacientes atendidos pela cirurgia vascular no HMM, no período entre abril de 2015 a setembro de 2019, sendo um total de 621 atendimentos.

A coleta de dados foi realizada no período de dois meses, de outubro a dezembro de 2019. Os dados foram disponibilizados pelo Serviço de Atendimento Médico e Estatístico (SAME) do hospital e através de busca ativa dos prontuários de pacientes internados no período especificado.

Os critérios de inclusão utilizados na pesquisa foram: pacientes atendidos pela clínica vascular e com diagnóstico de pé diabético, que realizaram tratamento clínico ou que evoluíram para procedimentos cirúrgicos. Foram excluídos atendimentos não relacionados ao pé diabético, além dos realizados em períodos distintos ao mencionado anteriormente.

As variáveis estudadas relacionaram-se aos tipos de procedimentos cirúrgicos, sexo e município de origem dos pacientes.

Os dados obtidos foram analisados e avaliados quanto a correlação com os pacientes diabéticos, especificamente aqueles que realizaram procedimentos cirúrgicos.

Posteriormente, foi confrontado os dados da análise regional com os encontrados na literatura, com o intuito de avaliar a relevância da implantação do serviço de referência ao paciente portador de pé diabético.

Os resultados foram tratados com recursos da estatística descritiva, para a apresentação em gráficos e percentuais. A estatística inferencial foi aplicada com os Testes G e Qui-Quadrado Aderência. O nível de significância adotado para a pesquisa, foi $\alpha=0.05$, ou $5 \%$.

Foram preservados os aspectos éticos em todas as etapas do estudo, em concordância com a Convenção de Helsinki, e de acordo com a Resolução nº 466/12 e 510/16 que trata das normas para pesquisas envolvendo seres humanos.

\section{Resultados}

Dos 621 atendimentos realizados pelo serviço de cirurgia vascular do HMM no período entre 2015 e 2019, 30\% foram realizados em pacientes com diagnóstico de fasceíte necrosante, $19 \%$ foram em pacientes com pé diabético, que realizaram apenas tratamento clínico. O restante, $51 \%$ dos atendimentos, corresponderam a procedimentos cirúrgicos de amputações, sendo a diferença estatisticamente significante em relação aos demais ( $p<0.0001)$, como demonstrado na Figura 1. 
Figura 1 - Atendimentos realizados pelo serviço de cirurgia vascular, HMM, 2015 a 2019.

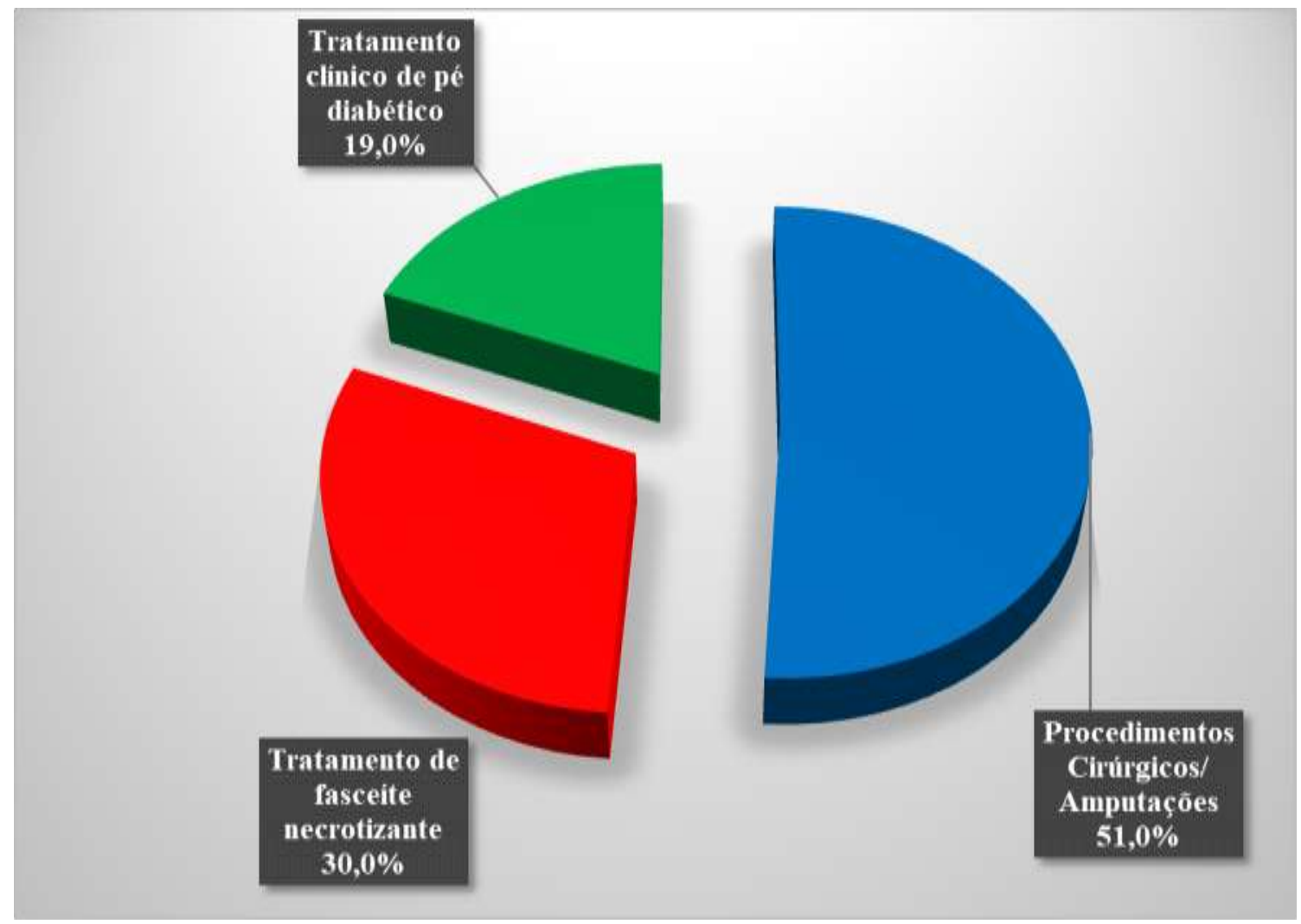

Fonte: SAME/HMM, *p < 0.0001 (Teste Qui-Quadrado Aderência).

Em relação a variável de gênero dos atendimentos realizados (Figura 2), observou-se que nos pacientes com diagnóstico de fasceíte necrosante, $59 \%$ dos indivíduos eram do sexo masculino e $41 \%$ do sexo feminino.

Nos pacientes, que realizaram apenas tratamento clínico do pé diabético, 50,4\% dos indivíduos eram do sexo masculino contra 49,6\% do sexo feminino. Quando comparada essa variável nos pacientes que evoluíram com procedimentos cirúrgicos, notou-se que $70 \%$ dos indivíduos eram do sexo masculino e $30 \%$ do feminino. O sexo masculino foi significativamente maior $(\mathrm{p}<0.0001)$ entre os grupos com fasceíte necrosante e daqueles que passaram por procedimento cirúrgico. 
Figura 2 - Atendimentos realizados no serviço de cirurgia vascular, HMM, 2015 a 2019.

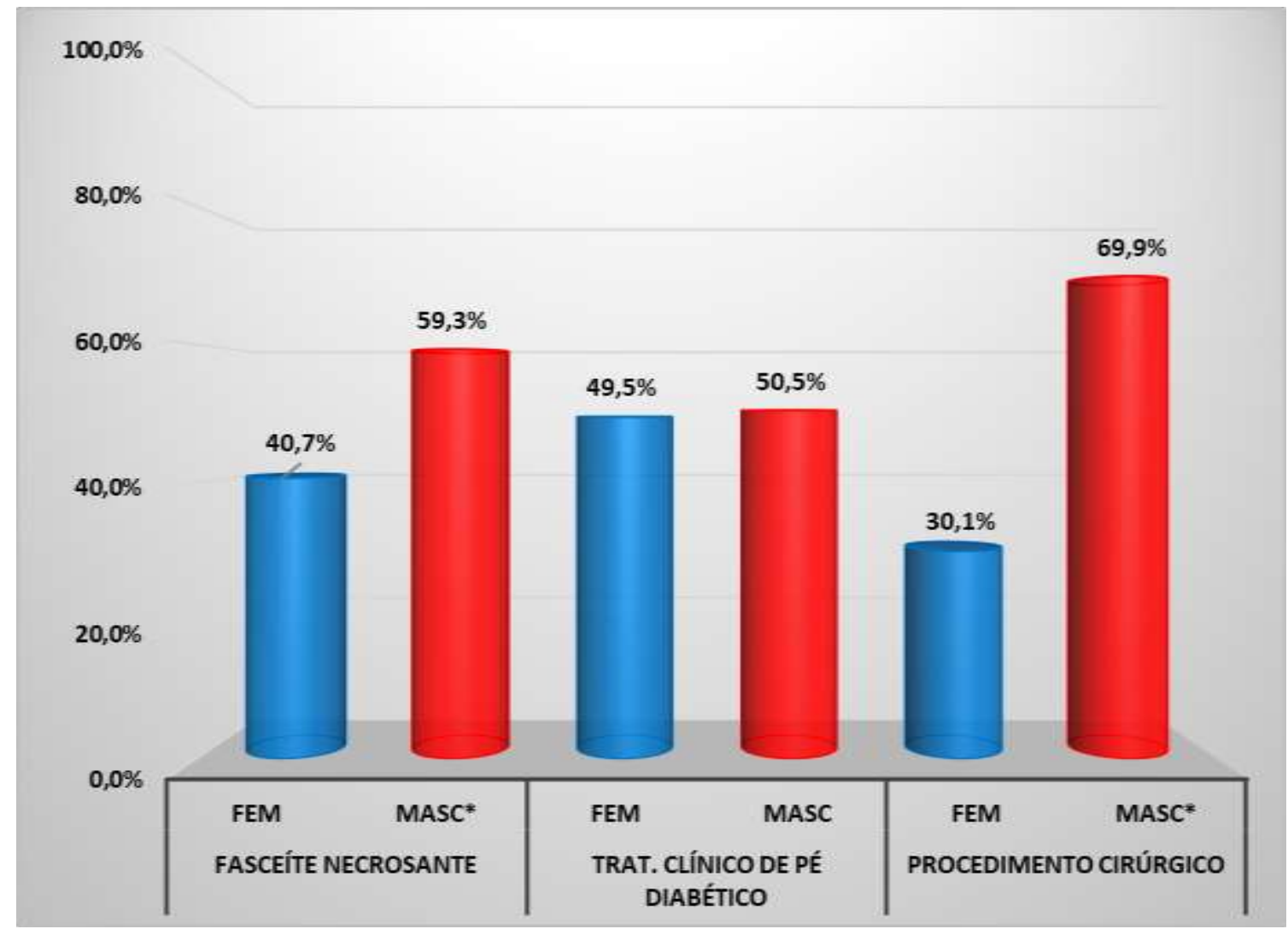

Fonte: SAME/HMM. *p=0.0001 Teste Qui-Quadrado Aderência.

Conforme mostra a Figura 3, os procedimentos cirúrgicos mais realizados pela cirurgia vascular no período avaliado foram de amputação de membros inferiores (47,4\%), seguido pela amputação de pododáctilos (45,2\%), em menor incidência a revisão de coto de amputação de pododáctilo $(4,4 \%)$ e revisão de coto de amputação de membro inferior $(2,8 \%)$. As amputações $(92.6 \%)$ foram o procedimento estatisticamente significante $(\mathrm{p}<0.0001)$, em relação aos demais. 
Figura 3 - Procedimentos cirúrgicos realizados, HMM, 2015 a 2019.

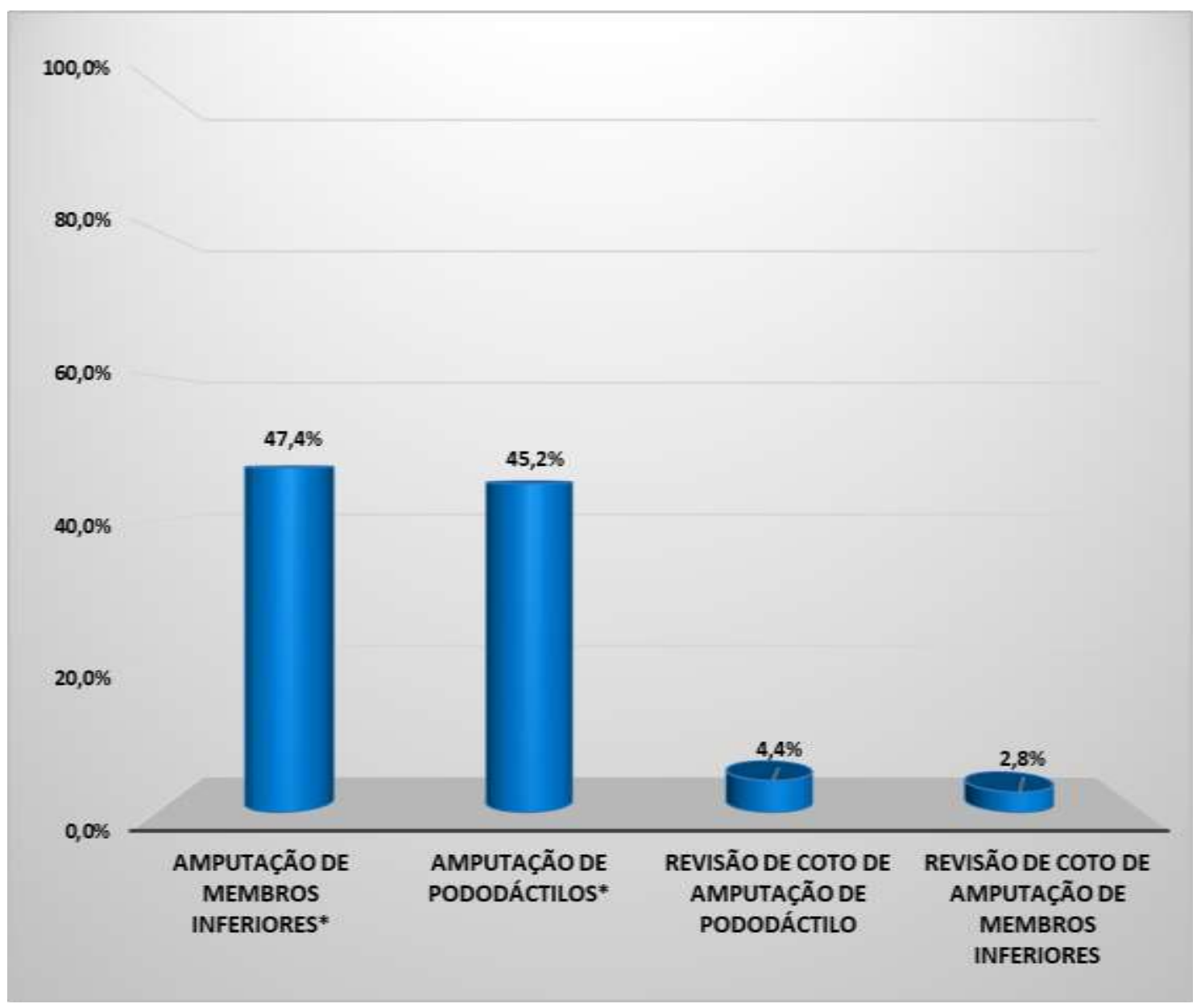

Fonte: SAME/HMM *p $<0.0001$ Teste G Aderência.

Considerando, os pacientes diabéticos que realizaram procedimentos cirúrgicos, foi observada uma prevalência dos indivíduos com esse diagnóstico de base. Dos 150 pacientes que realizaram cirurgia de amputação de membros inferiores, $75 \%$ tinham o diagnóstico confirmado; entre os indivíduos que realizaram amputação de pododáctilos, $80 \%$ deles eram diabéticos.

Nos indivíduos com diagnóstico de fasceíte necrosante se notou uma prevalência de $85 \%$ pacientes diabéticos. A proporcionalidade de pacientes diabéticos em relação aos não diabéticos foi estatisticamente significante nos 3 grupos avaliados ( $<$ 0.0001), como mostra a Figura 4. 
Figura 4 - Pacientes amputados ou portadores de fasceíte necrotizante segundo o diagnóstico de diabetes, HMM, 2015 a 2019.

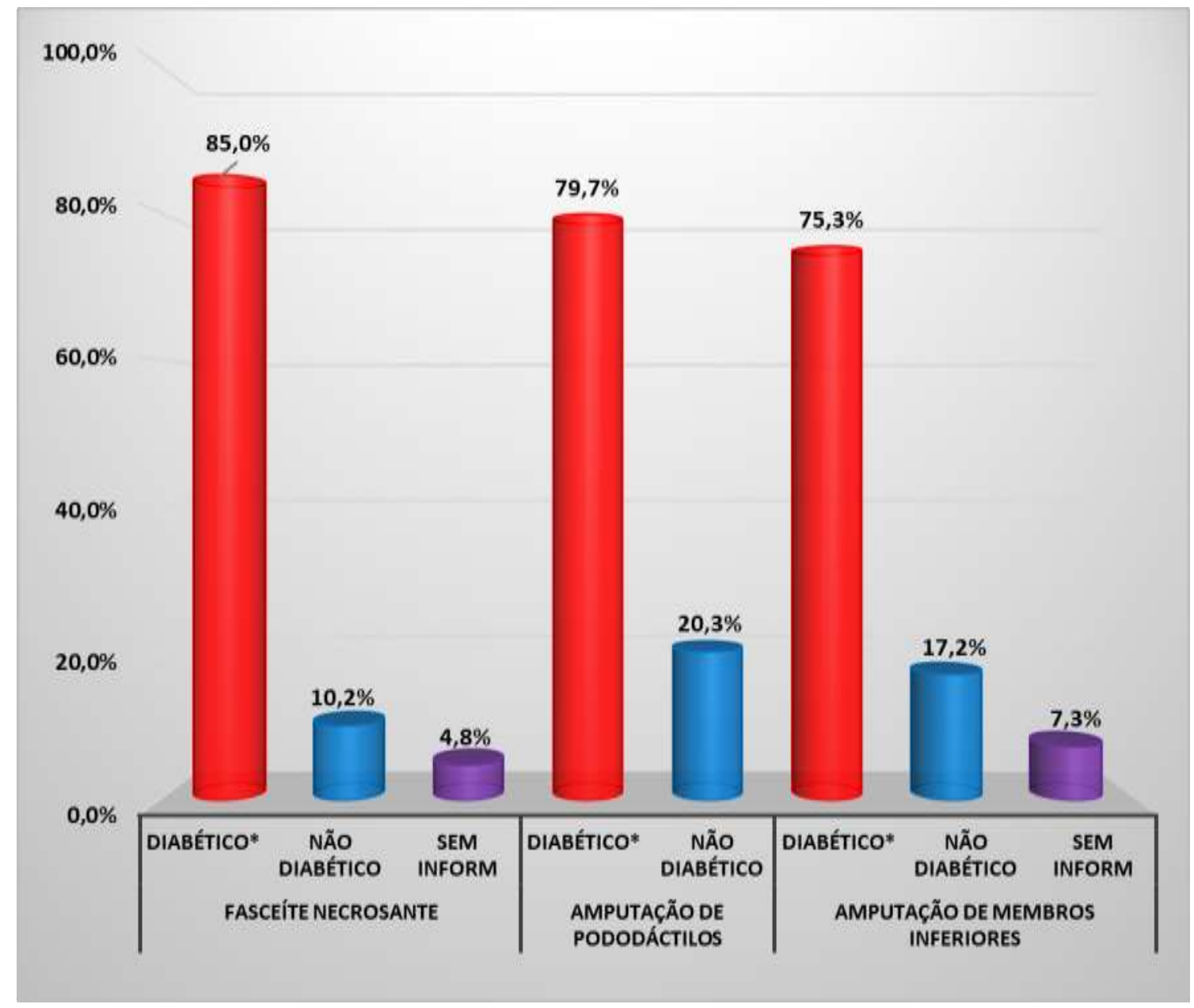

Fonte: SAME/HMM. *p $<0.0001$ Teste G Aderência.

Avaliando a cidade de origem dos pacientes atendidos no HMM, ficou evidente a dominância significante dos indivíduos de Marabá em relação a outros municípios ( $\mathrm{p}<0.0001$ ), sendo 85\% dos atendimentos realizados nesses pacientes. Outro município em evidência foi o de São João do Araguaia, com 5\% dos atendimentos. Demais cidades do estado identificadas no estudo foram Itupiranga, Ipixuna e Rondon do Pará, além de cidades de outros estados (Figura 5). 
Figura 5 - Municípios de origem dos pacientes, HMM, 2015 a 2019.

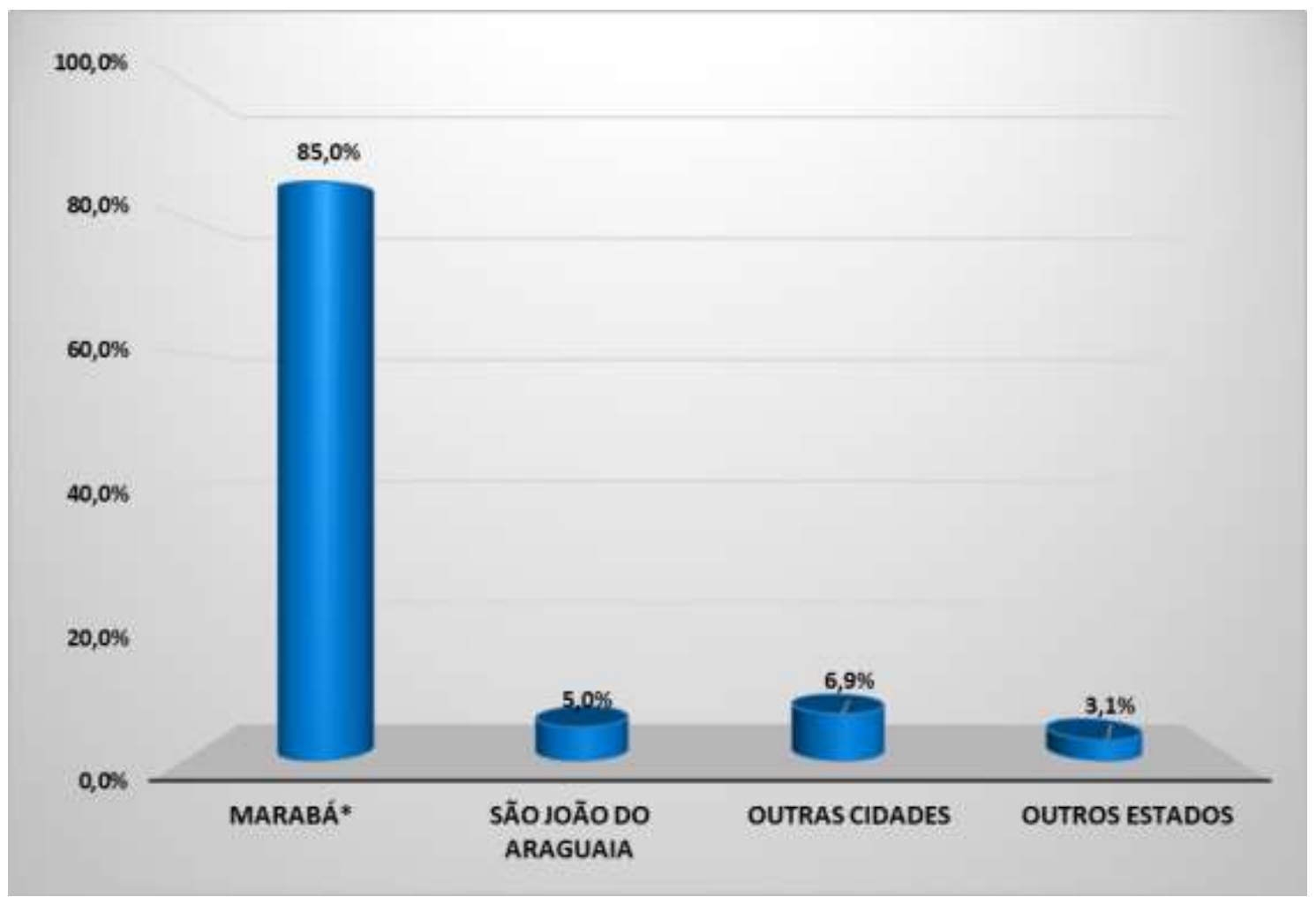

Fonte: SAME/HMM *p $<0.0001$ Teste G Aderência.

\section{Discussão}

Neste estudo foi verificado uma prevalência do sexo masculino. No estudo realizado em um hospital geral de Goiânia por Oliveira et al. (2016), apresentou dados similares aos encontrados nesta pesquisa, com uma igualdade nos casos de internações em pacientes diabéticos dos sexos masculino e feminino, entretanto com uma disparidade do sexo masculino quando avaliados o número de amputações entre esses pacientes.

Quando avaliado a incidência do DM entre os sexos, é consenso entre os estudos que os indivíduos do sexo feminino são prevalentes em relação ao masculino, contudo as complicações oriundas do diabetes são recorrentes e mais incidentes nos indivíduos do sexo masculino (Oliveira et al., 2016; Neto et al., 2017; Marques et al., 2018). Nessa conjuntura, Oliveira et al. (2016) afirmam que as mulheres possuem um maior conhecimento sobre o autocuidado com os pés, além de procurar e frequentar assiduamente os serviços de saúde. Diante disso, pode-se afirmar que as mulheres apresentam menos complicações da DM por se prevenirem mais que os homens e por possuírem uma visão mais holística da saúde e da sua importância para sua qualidade de vida.

Os resultados do presente estudo, demonstraram uma prevalência de procedimentos cirúrgicos nos atendimentos realizados pela equipe vascular do HMM. No período avaliado, 50,8\% dos atendimentos foram cirúrgicos e destes, observouse uma prevalência de pacientes com diagnóstico de diabetes mellitus, como representado nas Figuras 3, 4 e 5, onde se verificou que nos tipos de procedimentos cirúrgicos abordados, em todos os casos, os pacientes diabéticos eram em número superior. O Consenso Internacional sobre o Pé Diabético (Brasil, 2001), aponta que 40 a $60 \%$ de todas as amputações não traumáticas dos membros inferiores são em pacientes com diabetes e que é provável que a incidência de amputações relacionados a diabetes situa-se entre 5-24 casos para cada 100.000 habitantes/ano ou 6-8/1000 indivíduos diabéticos/ano.

Em virtude do alto índice de procedimentos cirúrgicos vasculares e a correlação com a diabetes, também evidenciado nesse estudo, o International Working Group on the Diabetic Foot - IWGDP (Schaper et al., 2017) define a diabetes como uma 
doença pandêmica e que cresce em nível alarmante, cerca de nove milhões de caso por ano. Entre suas principais complicações, o pé diabético promove um alto impacto negativo na qualidade de vida do indivíduo e sua família, além do seu elevado custo econômico e social do seu tratamento, especialmente quando evolui para uma amputação de membro.

As diretrizes de Sociedade Brasileira de Diabetes (SBD, 2016), relacionam as úlceras dos pés com casos de amputações, e apontam esta lesão como a principal causa de complicações em pacientes diabéticos nos países em desenvolvimento.

A cada 20 segundos uma pessoa perde um membro devido a DM, o que corresponde por ano, a um milhão de amputações de membros, em decorrência dessa doença (SBD, 2016).

Os dados encontrados no HMM corroboram com a literatura analisada. É possível perceber a relação dos atendimentos realizados pelo serviço vascular nesta instituição com as complicações oriundas do diabetes. Entre os atendimentos compilados, verificou-se uma incidência de $19 \%$ dos atendimentos em pacientes com diagnóstico de pé diabético e uma predominância de pacientes diabéticos nos procedimentos cirúrgicos realizados (amputações de membros inferiores $47,4 \%$, amputações de pododáctilos $45,2 \%$, revisão de amputação de pododáctilos $4,4 \%$ e revisão de amputação de membro inferior 2,8\%). Diante disso, é possível afirmar que as causas de complicações e amputações neste nosocômio estão intrinsicamente relacionadas ao Diabetes Mellitus.

Os custos médicos diretos anuais atribuídos a doença do pé diabético no Brasil em 2014 foram estimados em $\mathrm{R} \$ 361$ milhões, o que representa $0,31 \%$ das despesas de saúde pública no período avaliado, sendo do total 27,7 milhões (13\%) foram para internação e US \$333,5 milhões (87\%) para atendimento ambulatorial (Toscano et al., 2018).

Vislumbrando ainda o impacto socioeconômico do diabetes na nossa região. Epidemiologistas afirmam que 20-25\% das admissões hospitalares ocorrem por complicações da diabetes. Pacientes com úlcera de pé tem risco aumentado de amputação e maior taxa de mortalidade (Alavi et al., 2014; Martins-Mendes et al., 2014; Cardoso et al., 2018), o que pode ser agravado com a idade avançada, doença arterial periférica e anemia (Costa et al., 2017). Os custos hospitalares se tornam altos pelo tempo de hospitalização dos pacientes que evoluíram com amputações, variando entre 7 a 157 dias, com média de 41,7 dias. Quando internados em UTI a permanência média é de 22,8 dias (Oliveira et al., 2014).

O conhecimento sobre a procedência dos pacientes favorece uma reflexão sobre os fatores epidemiológicos dessa doença, auxiliando no desenvolvimento de medidas preventivas nas regiões mais acometidas. Por se tratar de um hospital de referência na região, o HMM atende toda uma população circunvizinha ao seu munícipio, como demostrado na Figura 5. Diante do exposto é possível evidenciar a necessidade da implantação dessas medidas na cidade de Marabá visto que 85\% dos atendimentos realizado neste hospital eram do seu próprio município. Somado a isso, há também a alta incidência de amputações realizadas em pacientes diabéticos em relação à outros estudo (Costa et al., 2017), demonstrando a necessidade de implantação de serviço de orientação e cuidados, com o objetivo de diminuir essa porcentagem.

A implementação do CRPDM tem como objetivo atuar na prevenção das complicações em pacientes com pé diabético e principalmente promover uma mudança da realidade no âmbito da sua atuação. O IWGDP recomenda a implantação de serviços básicos na comunidade, de ambulatórios ligados a hospitais ou centros especializados, de modo a se estabelecer, gradualmente, uma rede integrada para atendimento aos portadores de DM (Schaper et al., 2017).

A instituição de medidas educativas é fundamental para prevenção de forma eficaz das complicações nos pacientes diabéticos e a orientação com os pés é um importante instrumento na redução do risco de amputações de membros inferiores nesses indivíduos (Silveira et al., 2017).

A implementação de medidas conjuntas é indispensável para o alcance da prevenção das úlceras do pé diabético. A educação para as pessoas com diabetes, cuidadores e profissionais de saúde, desde atenção básica até a terciária, associada a implementação dos exames dos pés, podem apresentar excelentes resultados. Ainda neste contexto é preciso uma estruturação 
do serviço com o objetivo de alcançar as necessidades dos pacientes para um cuidado crônico em vez de intervenções agudas ou emergenciais (SBD, 2016).

A organização da rede de serviços dos pés de pessoas com DM deve levar em consideração a realidade local quanto a prevalência de diabetes, a capacidade técnica dos profissionais da rede e fluxos de atendimento iniciado preferencialmente nas unidades básicas de saúde (Brasil, 2016).

\section{Conclusão}

O presente trabalho apresentou a situação da saúde pública dos pacientes com DM atendidos no HMM, evidenciando uma realidade preocupante, caracterizada por altos índices de internações hospitalares, com 621 atendimentos realizados no período entre 2015 - 2019 pelo serviço de cirurgia vascular. As amputações foram apontadas como uma complicação do diabetes, sendo realizado 47,4\% de amputações de membros inferiores e 45,2\% de amputações de pododáctilos.

Nessa perspectiva, o CRPDM tem como objetivo implantar um serviço de referência para os pacientes diabéticos, baseado nos protocolos e recomendações do Ministério da Saúde, SBD e IWGDP. A perspectiva é mudar a realidade da cidade e região de Marabá, promovendo melhorias na assistência, com o intuito de diminuir as complicações e sequelas nessa população.

Futuros estudos devem ser realizados para verificar o nível de conhecimento dos pacientes sobre as medidas de controle e prevenção da diabetes e de suas complicações, pois a orientação e conscientização correta dos pacientes também podem contribuir para a diminuição das complicações advindas dessa enfermidade.

\section{Referências}

Alavi, A., Sibbald, R. G., Mayer, D., Goodman, L., Botros, M., Armstrong, D. G., Woo, K., Boeni, T., Ayello, E. A., \& Kirsner, R. S. (2014). Diabetic foot ulcers: Part I. Pathophysiology and prevention. Journal of the American Academy of Dermatology, 70(1), 1.e1-18.

Brasil. (2001). Grupo de Trabalho Internacional sobre Pé Diabético. Consenso internacional sobre pé diabético. Secretaria de Saúde do Distrito Federal.

Brasil. (2014). Ministério da Saúde. Caderno de Atenção Básica no 35. Estratégias para o Cuidado da Pessoa com Doença Crônica. Ministério da Saúde. https://www.dab.saúde.gov.br/portaldab/biblioteca.php?conteúdo=publicacoes/cab36.

Brasil. (2016). Ministério da Saúde. Secretaria de Atenção à Saúde. Departamento de Atenção Básica. Manual do pé diabético: estratégias para o cuidado da pessoa com doença crônica. Ministério da Saúde, Secretaria de Atenção à Saúde, Departamento de Atenção Básica. Ministério da Saúde.

Brasil. (2017). Diabetes aumenta no país e já atinge 9\% dos brasileiros. Ministério da Saúde. https://www.saude.gov.br.

Cardoso, N. A., Cisneros, L. D. L., Machado, C. J., Procópio, R. J., \& Navarro, T. P. (2018). Fatores de risco para mortalidade em pacientes submetidos a amputações maiores por pé diabético infectado. Jornal Vascular Brasileiro, 17(4), 296-302.

Costa, R. H. R., Cardoso, N. A., Procópio, R. J., Navarro, T. P., Dardik, A., \& de Loiola Cisneros, L. (2017). Diabetic foot ulcer carries high amputation and mortality rates, particularly in the presence of advanced age, peripheral artery disease and anemia. Diabetes \& Metabolic Syndrome: Clinical Research \& Reviews, 11, S583-S587.

Estrela, C. (2018). Metodologia Científica: Ciência, Ensino, Pesquisa. Editora Artes Médicas

Girach, A., Manner, D., \& Porta, M. (2006). Diabetic microvascular complications: can patients at risk be identified? A review. International Journal of Clinical Practice, 60(11), 1471-1483.

Gonçalves, R. R, de Moura, N. O. (2019). Diabetes mellitus e suplementação de proteínas e fibras: uma revisão de literatura. Revista de Iniciação Científica da Universidade Vale do Rio Verde, 8(2), 1.

Guariguata, L., Whiting, D. R., Hambleton, I., Beagley, J., Linnenkamp, U., \& Shaw, J. E. (2014). Global estimates of diabetes prevalence for 2013 and projections for 2035. Diabetes Research and Clinical Practice, 103(2), 137-149.

Karami-Mohajeri, S., \& Abdollahi, M. (2011). Toxic influence of organophosphate, carbamate, and organochlorine pesticides on cellular metabolism of lipids, proteins, and carbohydrates: a systematic review. Human \& experimental toxicology, 30(9), 1119-1140.

Marques, A. D. B., Silva, L. M. S., Moreira, T. M. M., \& Torres, R. A. M. (2018). Associação entre internação hospitalar por diabetes mellitus e amputação de pé diabético. Enfermeira Global, 5, 25248-25257. 
Research, Society and Development, v. 10, n. 6, e12810615586, 2021

(CC BY 4.0) | ISSN 2525-3409 | DOI: http://dx.doi.org/10.33448/rsd-v10i6.15586

Martins-Mendes, D., Monteiro-Soares, M., Boyko, E. J., Ribeiro, M., Barata, P., Lima, J., \& Soares, R. (2014). The independent contribution of diabetic foot ulcer on lower extremity amputation and mortality risk. Journal of Diabetes and its Complications, 28(5), 632-638.

Neto, M. O., Pereira, M. S., Pinto, M. A. H., Agostinho, L. M., Reinaldo Junior, F. E., \& Hissa, M. N. (2017). Avaliação do autocuidado para prevenção do pé diabético e exame clinico dos pés em um centro de referência em diabetes mellitus. Journal of Health and Biological Sciences, 5(3), $265-271$.

Oliveira, A. F., De Marchi, A. C. B., Leguisamo, C. P., Baldo, G. V., \& Wawginiak, T. A. (2014). Estimativa do custo de tratar o pé diabético, como prevenir e economizar recursos. Ciência \& Saúde coletiva, 19(6), 1663-1671.

Oliveira, J. C., Taquary, S. A. S., Barbosa, A. M., \& Veronezi, R. J. B. (2016). Pé diabético e amputações em pessoas internadas em hospital público: estudo transversal. ABCS Health Sciencec, 41(1), 34-39.

Santos, M. M. M. (2017). Prática do autocuidado com os pés na prevenção das complicações neurológicas periféricas das pessoas com Diabetes Mellitus [Trabalho de Conclusão de Curso - Monografia]. Centro de Educação e Saúde, Universidade Federal de Campina Grande.

Schaper, N. C., Van Netten, J. J., Apelqvist, J., Lipsky, B. A., \& Bakker, K. (2017). Prevention and management of foot problems in diabetes: a summary guidance for daily practice based on the 2015 IWGDF guidance documents. Diabetes Research and Clinical Practice, 124, 84-92.

Silveira, D. M., Ferreira, L. V., Fraga, G. H. W. S., \& Costa, M. B. (2017). Pé Diabético: onde podemos intervir? HU Revista, 43(1), 13-18.

Sociedade Brasileira de Diabetes - SBD. (2016). Diretrizes da Sociedade Brasileira de Diabetes (2015-2016). A.C. Farmacêutica.

Toscano, C. M., Sugita, T. H., Rosa, M. Q. M., Pedrosa, H. C., Rosa, R. S., \& Bahia, R. L. (2018). Annual Direct Medical Costs of Diabetic Foot Disease in Brazil: A Cost of Illness Study. International Journal of Environmental Research and Public Health, 15(1), 1-13. 